\title{
A Study of Human Performance in Recognizing Expressive Hand Movements
}

\author{
Ali-Akbar Samadani, Brandon J DeHart, Kirsten Robinson, Dana Kulić, Eric Kubica, Rob Gorbet
}

\begin{abstract}
This paper presents a study on human performance in recognizing affective expressions conveyed through movements of hand-like structures. One movement sequence, closing and opening the hand, was performed by a demonstrator in 3 sets of 5 repeated trials, each set intending to convey a different affective expression. Three different expressions, sadness, happiness and anger, were considered. Expressive movement animations were replicated with a human-like hand model, a stick hand model and with a model resembling a palm frond structure. The structures tested have identical kinematics but different physical appearance. The ability of a human to correctly identify the intended expressive movements performed on these different structures was tested with 66 users viewing videos of the animated structures and reporting via an online questionnaire. Results show that anger is reliably perceived by observers from animated movements on different structures, while the other emotions are easily misperceived. The physical appearance of the structure has some impact on perception performance, but was not found to be statistically significant in this study. Furthermore, analyzing the participants' responses in the context of the valence-arousal model of emotion shows that the subjects were able to recognize the arousal component of the affective hand movements across all structures.
\end{abstract}

\section{INTRODUCTION}

$\mathrm{T}$ he design of responsive environments is an evolving field in architectural practice, which involves the development of "intelligent" elements and structures that perceive human presence and respond utilizing an integrated design of sensors, artificial intelligence and actuators. Actuated response can take various forms (e.g., motion, sound, light, heat) and may be applied in a localized area or globally. Recently, a series of such environments demonstrating localized and global kinetic response to occupants has raised the question of whether humans might recognize expressed emotions when these are displayed using a kinetic mechanism.

While there is extensive work on producing expressiveness in humanoid robots ([1], [2], [3]) there is little that looks at generalizing human-like motion to other structures and mechanisms that could be integrated into responsive environments. A few notable examples of evocative designs at the boundary of robotics and expression

Ali-Akbar Samadani, Brandon J DeHart, Kirsten Robinson, Dana Kulić and Eric Kubica are with the Electrical and Computer Engineering Department at University of Waterloo, Waterloo, ON N2L 3G1, CANADA, (phone: 519-888-4567 ext 31474; e-mail: asamadan|bjdehart|k4robinson |dkulic|ekubica]@uwaterloo.ca, respectively).

Rob Gorbet is with the Centre for Knowledge Integration at University of Waterloo, Waterloo, ON N2L 3G1, CANADA(e-mail: rbgorbet@ uwaterloo.ca). are Theo Jansen's "Strandbeest" species, which use six-bar legs powered by the wind to roam the beach [4]; the woven installation, Strange Metabolisms, by Mette Ramsgard Thomsen and Toni Hicks that curls and extends in response to its surroundings [5]; and BMW's GINA car concept, which is a shape shifting car covered with a Lycra fabric skin incorporating many actuators to control and adapt the body form to driving conditions, as well as giving life-like attributes to the car such as eyelid-like headlights [6]. These structures, while evocative, have not been studied explicitly in terms of how users perceive them.

The importance of studying human motion perception stems from the fact that humans infer and ascribe affective meaning to observed motions and gestures, even those performed by non-anthropomorphic structures [7], [8], [9], [10], [11]. Moreover, the human affective state is a fundamental component of human-human interaction, which should be taken into consideration when developing interactive human-machine systems; interactions without affective state consideration are often perceived as "cold, incompetent and socially inept" [12].

There is significant work on the human perception of whole body movement and postures. Camurri and his colleagues [8] tested human movement perception in four emotional categories of anger, fear, grief and happiness conveyed through the same dance movement. They report that the human observers were able to detect the emotions transmitted through the dance movement. McDonnell et al. [9] investigated the human perception of movements conveying the 6 basic Ekman emotions [13] displayed on virtual replicates of an actor (high and low resolution virtual human counterparts, a wooden mannequin, a cartoon character and a zombie-like character), along with the effect of the replicates' structures on human perception. The participants were able to recognize basic emotions displayed on different characters, with body type affecting the rated emotion in some cases. In [14], it is shown that the human perception of full body motion is affected by the shape of the animated structure and by the geometric model used for rendering. In [8] and [11], different body movements and postures are associated with different emotional states.

Fewer works focus on affect perception from hand and arm movements alone, even though there is evidence that these movements are most significant for distinguishing between emotions [11]. In a recent study [15], it is found that human observers are sensitive to very subtle errors in finger motions (of virtual human characters), such as a synchronization error of 0.1 seconds. It is also found that the perception of finger motion can change the interpretation of 
the entire displayed scene. In another study [45], it is reported that the underlying emotions conveyed during Finnish sign language communication were recognized by those having no knowledge of the language itself. Furthermore, neuroscientists report engagement of different perceptual and visuomotor processes when observing actions demonstrated on virtual hand-like structures as compared to real hand movements [16], [17], [18].

These studies suggest that physical postures and movements can convey at least basic emotions and that the physical appearance of the structure demonstrating the movement may play a role in human perception of emotion.

Integrating emotion display and recognition is a question mostly confronted by the researchers in social robotics and assistive devices. Numerous expressive robots have been built, mostly for facial and full body motion [19], [20], [21]. In the majority of these works, hand gestures are used as complements to face and body expressions and less attention has been directed to the expressions conveyed by hand movements alone. Work on isolated hand-like mechanisms has focused primarily on manipulator tasks and control interfaces [22]. Where gestures have been considered explicitly, the emphasis has been on communicative gestures such as those used in sign languages, or on the use of hand motion to control computers and musical instruments [23].

Human gestures can be subtle and are understood by humans sometimes without being consciously noticed and often without explicit consciousness of the features that communicate expressions [24]. Gestures sometimes even communicate the information that people intend to hide (e.g., negative body-language). It is unlikely, therefore, that respondents can tell us precisely how to generate the full range of communicative gestures. Furthermore, human gestures are highly variable: timing, hand shapes, and the flexibility of hands all vary across subjects. Motion capture technologies make it possible to record and reproduce motions precisely to be used in examining what is conveyed by a gesture.

One way to characterize motion is through the use of the low-level variables such as position, velocity, and acceleration. Expression can also be treated at a higher level of abstraction. High level descriptions of motion come from research on human movement in the humanities and performance studies [25], psychology [24], and biomechanics [26]. Rodolf Laban's Theory of Effort is one of the prominent examples of the research on human movement [25]. Laban notation was developed as a way of writing and analyzing dance choreography [27]. Laban effort is a measure of the dynamics and expressiveness of a movement. For instance, both punching someone in anger and reaching for a glass have similar movement kinematics involving the extension of the arm. However, they are very different in terms of the strength, timing, and control of the movement. Research on automated generation and computational analysis of Laban notation has been carried out for applications in dance choreography [28], [29] and in human-machine interaction [30]. The low-level movement characterization is reported to be in direct association with the strength, timing, and control of the movement described by Laban components in [31] and [8]. For instance, fast tempo is associated with anger and happiness while a slow tempo is associated with sadness. In this work, Laban effort is used as a basis for demonstration of the expressive movements studied.

The research in this paper is motivated by the development of a series of architectural responsive environments, called the Hylozoic series (e.g., [32], [33]). The geotextile-like support meshwork is layered with responsive mechanisms that allow them to create subtle motions giving the impression that the environment is "sensitive" and perhaps even capable of expressing emotions. These environments are constructed of massively repeating custom laser-cut components, microprocessors, sensors and actuators (Fig. 1). The responsive components consist of layers of mechanical fronds and whiskers (Fig. 1.b) that move in response to the human occupants. The motivation for our research comes from observing the varied emotional reactions that occupants have to the mechanisms and motion in the space, and wondering whether one could achieve a more consistent and deliberate affective expression. To attempt to do so requires understanding how occupants perceive the structure's motion, and what influences the structure's physical appearance might have on that perception. We hope to identify those features of nonlife-like kinetic display mechanisms, and their movements, that are capable of communicating specific human expression.
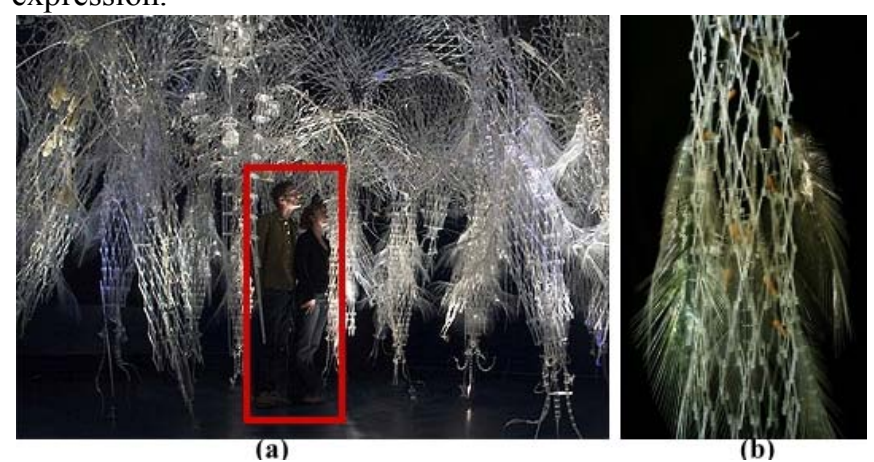

Fig. 1. a) Two visitors immersed in Hylozoic Soil (highlighted with a red outlined rectangle), a responsive architectural geotextile environment [33]. Reprinted with permission. b) Hylozoic Soil consists of layers of mechanical fronds and whiskers that move in response to the human occupants [33]. Reprinted with permission.

In this paper, we consider the affective hand movements in isolation from the body and generalize to other hand-like structures with non-anthropomorphic physical appearance. The rationale for choosing hand movements in this study is that the hand is an important medium for communicative gestures [43] and it closely resembles the motion style and structure of the moving components of the Hylozoic environments. In the present work, three different hand-like structures were used: an anthropomorphic model (most human-like structure, left-most structure in Fig. 2), a stick 
model (middle structure in Fig. 2), and a less hand-like structure composed of palm fronds (right-most structure in Fig. 2). All three structures have the same kinematics, while the outer physical appearance differs. The use of the frond hand-like structure is motivated by mechanical frond components of the Hylozoic series (Fig. 1.b). Three different expressions considered here are: sadness, happiness and anger. The recognition rate of the intended emotion of a movement, while watching a video of each of the movements, is evaluated through an online questionnaire. Furthermore, the impact of physical appearance of the structures (human-like vs. non-human like) on emotion recognition is investigated.

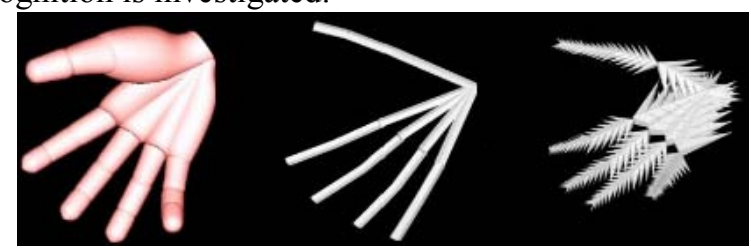

Fig. 2. Structures used to display expressive movements. From left to right: human-like hand model, stick hand model, frond like structure

Work on the capacity of people to recognize expression in non-human-like structures can offer insight into what motion features convey expression and whether the physical appearance of the display structure affects the perception of the movement. Our long term goal is to design new mechanisms to generate an approximation of expressive human motion, while maintaining recognizable expressive content, yielding a kinetic environment that is engaging and comprehensible.

To the knowledge of the authors, this work is the first attempt in studying human perception of expressive movement demonstrated on different hand-like structures. This paper is organized as follows: In Section II, an overview of the methodology used in this work is presented followed by data analysis and results in Section III. Section IV discusses the results and directions for future work and Section V provides conclusions.

\section{Methodology}

In this study, the ability of a human to correctly identify emotions conveyed through movement displayed on humanlike and non-human-like structures is studied. A single movement sequence, closing and opening the hand, mainly consisting of phalangeal and carpo-metacarpal joint movements, is used in 3 sets of 5 trials, where each set conveys a different expression. Three different expressions are considered: sadness, happiness and anger. A demonstrator, who has been exposed to Laban notation, and was familiar with other human movement perception works (e.g. Camurri et al [8]), performed the hand movements while wearing a Dataglove (ShapeHand from Measurand) [34], [41]. Similar practices in data collection for studying human perception of expressive movements are reported in the [8]. The resulting angry and sad movements were characterized by high and low tempo, respectively. A happy movement sequence was characterized by fast tempo as well as multiple changes in velocity and direction. Videos of these movements are available in reference [44]. Local Euler angles for the root joint (i.e. wrist) and three joints A, B and $\mathrm{C}$ along each finger (Figure 3 ) were collected at $\sim 84$ frames per second and subsequently used to animate the movement on three different hand-like models: an anthropomorphic model, a stick model, and a less hand-like structure composed of palm fronds (Fig. 2). The use of a single demonstrator in this study obviates the need for movement standardization across demonstrators.

In the online questionnaire, videos of the movements performed on the three different structures were shown to the participants. They were then asked to evaluate the demonstrated movements. A total of 66 participants $(26.8$ years \pm 4.8 years, 38 male and 28 female) completed the survey. Participants were healthy adults with a basic working knowledge of computers and were recruited online via social media tools such as email and Facebook. They were provided with detailed information about the study and the procedure to complete the online questionnaire. The study received ethics approval from the Office of Research Ethics, University of Waterloo, and a consent form was signed electronically by each participant prior to the start of the questionnaire.

Three different survey versions, each including 6 videos, were used (Table I). The 66 participants were divided into three groups, each taking one version of the survey. The following naming format is used to refer to the animations in this paper: "structure_intended expression" (e.g., "Real_Happy" represents the happiness emotion displayed on the human-like hand model). Figure 3 shows video frames for a Real_Angry movement. The videos of expressive hand movements were shown in a randomized order. Each video was accompanied by two questions. The first question was a multiple-selection question asking participants to select among a list of keywords those that most closely described the animated structure in the video. The keywords were: Human-like, robot-like, organic, artificial, hand-like, plant-like, stick-like, represents biological motion, represents mechanical motion, animallike, paw-like, hoof-like, friendly, unfriendly, cartoon-like, pleasant, unpleasant, aggressive, gentle, and none.

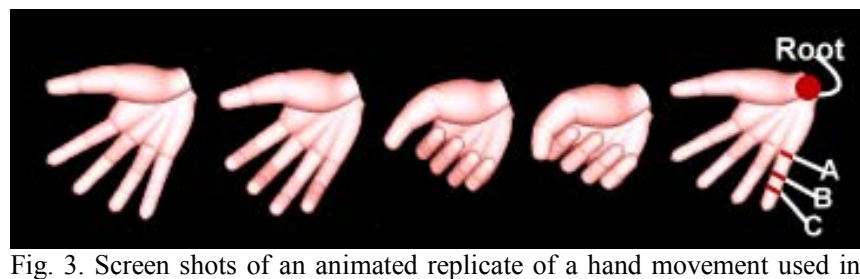

Fig. 3. Screen shots of an animated replicate of a hand movement used in this study. Local Euler angles were collected for the wrist (root) and three joints (A, B, and $\mathrm{C}$ ) along each finger. Joints in each figure are named as shown on the little finger of the far right hand (e.g. in index finger, A: proximal joint, B: intermediate joint, $\mathrm{C}$ : distal joint). 
TABLE I

3 SURVEY VERSIONS EACH CONTAINING 6 VIDEOS OF EXPRESSIVE MOVEMENTS.

\begin{tabular}{ccc}
\hline \hline Version 1 & Version 2 & Version 3 \\
\hline Real_Angry & Real_Happy & Real_Angry \\
Real_Happy & Real_Sad & Real_Sad \\
Stick_Angry & Stick_Angry & Stick_Happy \\
Stick_Happy & Stick_Sad & Stick_Sad \\
Frond_Happy & Frond_Angry & Frond_Angry \\
Frond_Sad & Frond_Happy & Frond_Sad \\
\hline \hline
\end{tabular}

The second question asked the subjects to rate on a Likert scale the extent to which each one of the 6 Ekman basic emotions [13] was conveyed in the displayed animation, with 1 being "not conveyed at all" and 6 being "strongly conveyed". We used all 6 Ekman emotions in the questionnaire to determine emotion recognition capabilities accurately. Offering participants the choice of 6 emotions gives a more accurate picture of recognition rate, since it does not artificially constrain the responses and shows whether emotions are unambiguously recognized.

Only responses with complete answers for each individual video were considered for analysis. Total numbers of 33, 36, 34, 37, 33, 33, 33, 36, and 33 responses for Real_Angry, Real_Sad, Real_Happy, Frond_Angry, Frond_Sad, Frond_Happy, Stick_Angry, Stick_Sad, and Stick_Happy, were collected respectively.

\section{DATA ANALYSIS}

\section{A. Structure description}

First, participants' responses to the structure description keywords were assessed. Table II shows the descriptors most commonly selected by participants; only keywords selected by more than $45 \%$ of the respondents are shown.

TABLE II

PERCENTAGE (\%) OF MOST FREQUENTLY (OVER 45\% OF THE CASES) CHOSEN DESCRIPTORS FOR DIFFERENT STRUCTURES AND INTENDED

\begin{tabular}{|c|c|c|c|c|}
\hline Structure & Happiness & Sadness & Anger & $\begin{array}{l}\text { overall } \\
\text { affects }\end{array}$ \\
\hline Real & Friendly:47 & $\begin{array}{l}\text { Gentle: } 89 \\
\text { Friendly:72 }\end{array}$ & $\begin{array}{l}\text { Aggressive:58 } \\
\text { Unfriendly:48 } \\
\text { Unpleasant: } 45\end{array}$ & $\begin{array}{c}\text { Human-like:73 } \\
\text { Hand-like:85 }\end{array}$ \\
\hline Stick & - & $\begin{array}{l}\text { Gentle:50 } \\
\text { Friendly:47 }\end{array}$ & $\begin{array}{l}\text { Aggressive:61 } \\
\text { Unfriendly:58 } \\
\text { Unpleasant:61 }\end{array}$ & $\begin{array}{c}\text { Hand-like:56 } \\
\text { Artificial:62 } \\
\text { Stick-like:56 }\end{array}$ \\
\hline Frond & $\begin{array}{l}\text { Unpleasant:48 } \\
\text { Unfriendly:54 }\end{array}$ & - & $\begin{array}{l}\text { Aggressive:76 } \\
\text { Unfriendly:70 } \\
\text { Unpleasant:62 }\end{array}$ & $\begin{array}{c}\text { Hand-like:54 } \\
\text { Plant-like:60 } \\
\text { Unfriendly:54 } \\
\text { Unpleasant:55 } \\
\text { Aggressive:52 }\end{array}$ \\
\hline $\begin{array}{l}\text { Overall } \\
\text { structures }\end{array}$ & - & - & $\begin{array}{l}\text { Aggressive: } 65 \\
\text { Unfriendly:59 } \\
\text { Unplesant:56 } \\
\end{array}$ & - \\
\hline
\end{tabular}

This analysis revealed that the human-like structure was the one described most frequently as human-like, regardless of the displayed expression. All of the structures were frequently described as hand-like. The stick model structure was most often described as artificial and stick-like, while the frond structure was described as plant-like, aggressive, unfriendly, and unpleasant. The observation that the frond structure was described as plant-like was expected while associating negative descriptive keywords to this structure is probably due to the shape of the structure, which has many thorn-shaped elements covering the surface of the hand. As can be seen in Table II, there is also an interaction between the assessment of the structure and the emotion displayed. For instance, all structures demonstrating the anger expression are commonly described as aggressive, unfriendly, and unpleasant. For the sad movement, the stick and the human-like structures were frequently described as gentle and friendly, while this descriptor was not commonly ascribed to the frond structure, which was described as gentle and friendly in only $22 \%$ and $34 \%$ of the cases. For happy movements, the human-like structure were described as friendly in $47 \%$ of the responses, while this attribute was not ascribed to the stick and frond structures.

\section{B. Emotion Rating}

A bar chart representing the frequencies of different emotion ratings for each structure-affect animation pair, averaged among the participants was obtained. Figures 4, 5 and 6 show the average emotion rating along with the standard error (SE) for the expression demonstrated on the human-like, stick and frond structures, respectively. As can be seen in these figures, the anger expression is consistently recognized regardless of the structure. The happiness expression is not recognized in any of the structures (see Fig. 4 for Real_Happy). It is observed that in the cases of the stick and frond structures, the anger emotion is rated relatively high, whereas for the human-like structure, the happy movement is closely rated as surprise, fear and happy.
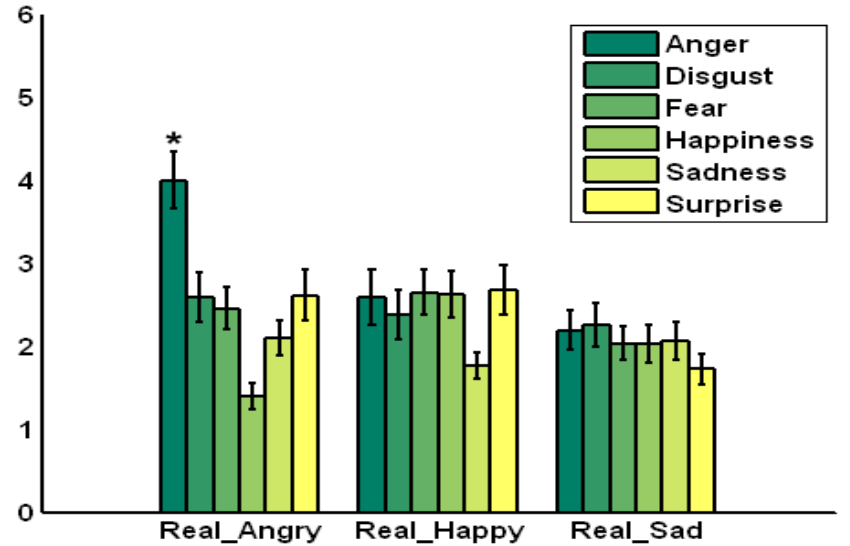

Fig. 4. Average emotion rating (mean \pm SE) for expressions displayed on the human-like hand model. "*” indicates significant difference in ratings of the intended emotion versus other emotions at $p<0.05$.

For each emotional category, statistical analysis using a two-way ANOVA was performed separately for all the participants' ratings and animations. In this test, the main effect of the physical appearance and the intended emotion as well as the interacting effect of these two factors on the ratings for each emotion was analyzed. Our first factor is the 
physical appearance and the second factor is the intended emotion. In all instances, differences were considered significant at $p<0.05$.

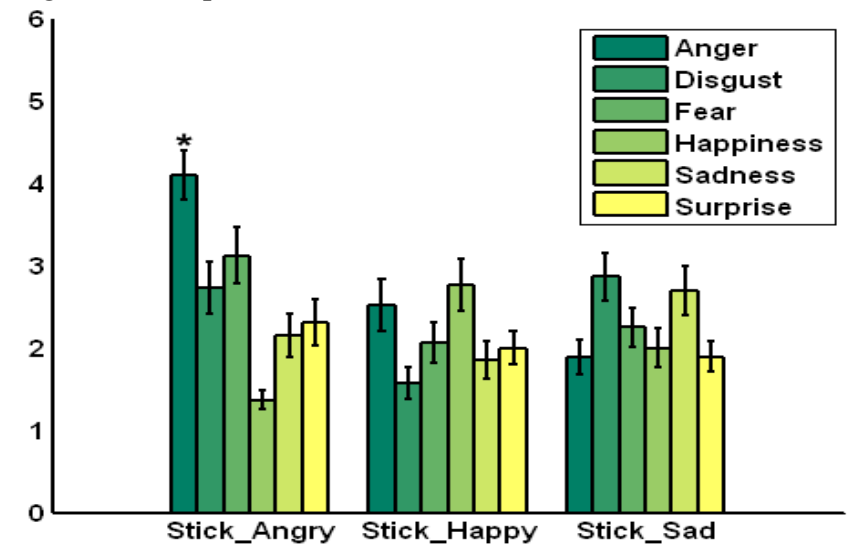

Fig. 5. Average emotion rating (mean $\pm \mathrm{SE}$ ) for expressions displayed on the stick-hand model. "*” indicates significant difference in ratings of the intended emotion versus other emotions at $\mathrm{p}<0.05$.

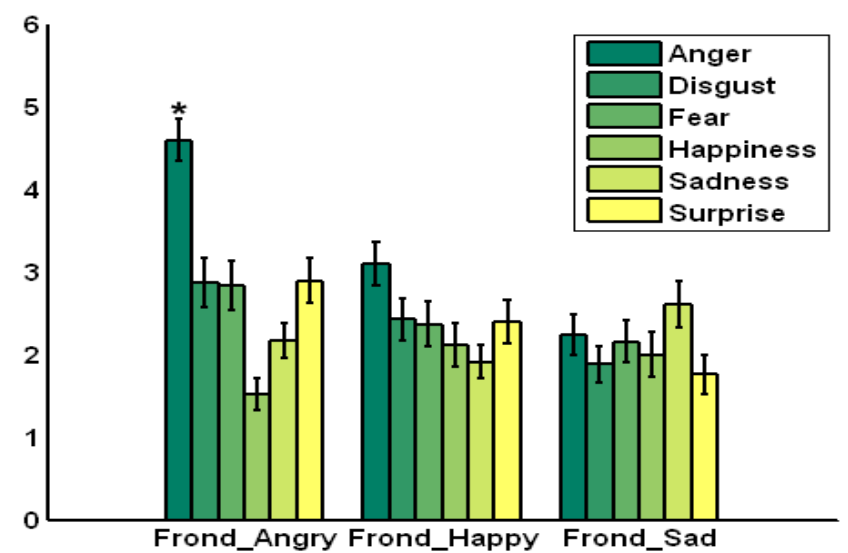

Fig. 6.Average emotion rating (mean \pm SE) for expressions displayed on the frond-hand model. "*” indicates significant difference in ratings of the intended-emotion versus other emotions at $\mathrm{p}<0.05$.

Table III shows the $p$-values obtained for the ratings of different animations for anger, happiness, and sadness. As can be seen in this table, the intended-emotion factor has a main effect on the rating of all the emotions (Anger, Happiness, and Sadness). This can be interpreted as follows: the intended-emotion, which is characterized by specific movement qualities [9] for different expressive movements, was a critical factor in evaluating the expression in different animations. Considering a significance level of $p<0.05$, the physical appearance does not show any effect on the emotion ratings. This result indicates that humans make use of movement cues associated with different emotions with little regard to the physical appearance of the animated structure.

In addition, the two-way ANOVA results reject the interaction effect of physical appearance and intendedemotion in all instances. This means that the impact of one factor does not depend on the level of the other. For instance, the rating of sadness in the Real_Angry animation is generated by considering the movement qualities alone; the appearance of the human-like hand model makes no significant contribution to the rating.

TABLE III

$P$-VALUES FROM TWO-WAY ANOVA TEST “*” SIGN INDICATES A SIGNIFICANT DIFFERENCE

\begin{tabular}{lccc}
\multicolumn{4}{c}{ “* SIGN INDICATES A SIGNIFICANT DIFFERENCE } \\
\hline \hline & Anger & Happiness & Sadness \\
\hline Physical appearance & 0.154 & 0.456 & 0.388 \\
& $0.000^{*}$ & $0.000^{*}$ & $0.001^{*}$ \\
Intended-Emotion & 0.5594 & 0.655 & 0.440 \\
$\begin{array}{l}\text { Physical appearance } \times \\
\text { Intended-Emotion }\end{array}$ & & & \\
\hline \hline
\end{tabular}

Table IV shows the confusion matrix for the recognition of anger, happiness, and sadness emotions demonstrated on the human-like (real), stick (stick), and frond-like (frond) structures. An emotion is considered recognized if it is rated 3 or above on the Likert scale. Structure differences are observed in the perception errors. For example, both the happiness and the sadness movement are more likely to be perceived as anger on the frond structure than on the other two structures.

TABLE IV

CONFUSION MATRIX SHOWING PERCENTAGE (\%)* OF EMOTION RATING FOR EACH INTENDED-MOTION DISPLAYED ON DIFFERENT STRUCTURES.

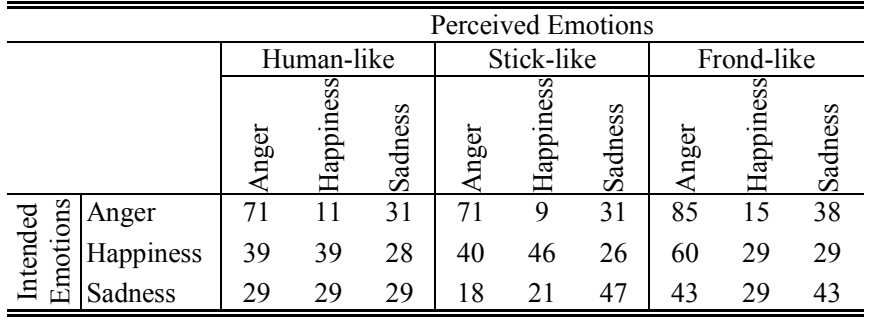

* Since participants were asked to rate all 6 Ekman emotions on a 6 point Likert scale, there are cases where an expressive movement was rated 3 or above for more than one emotion. On the other hand, there are cases in which anger, happiness and sadness were all rated below 3 . This is why none of the emotion ratings add up to $100 \%$ in the confusion matrix.

In addition, a pair-wise student t-test was performed between the participants' rating of the intended-emotion in each animation versus the other emotions to determine the significance of the rating of the intended-emotion. For instance, in the case of the Real_Angry animation, we want to check if the anger rating is significantly different than other ratings at $p<0.05$. It is found that the rating for anger is significantly different in all cases in which the intendedemotion was anger (see Figs. 4, 5, 6). The $p$-values obtained for the Real_Happy animation show that a significant difference only exists between the happiness and sadness emotion ratings. In the case of Stick_Happy, other than the anger rating, the rating for the happiness emotion is found to be significantly different than other emotions. In Frond_Happy, only the rating for the angry emotion is significantly different than the happiness rating and it appears that the hand movement is misrecognized as anger. In this case, the rating for anger is significantly higher than the ratings for all the other emotions. No significant 
difference between the rating for sadness and the other emotions is found in the case of Real_Sad. Sadness ratings for the Stick_Sad emotion are significantly different than anger, happiness and surprise ratings, but there is no significant difference with disgust and fear. For Frond_Sad the rating for sadness is significantly higher than surprise, disgust and fear, but no significant difference is observed between the sad rating and ratings for happiness and anger. Intended-emotions with a significantly higher rating than all other emotions are indicated by "*” on the bars in Figures 4, 5 , and 6 .

\section{Valence-Arousal Based Analysis}

The results from Section III.B indicate poor recognition capabilities in the discrete Ekman emotion categories. An alternative way to represent emotions is by lower dimensional models of affect ([38], [39], and [40]). The circumplex model divides the affective state space into two fundamental dimensions: valence and arousal [38]. Discrete emotion categories can be mapped to this two-dimensional model (e.g., sadness is an emotion characterized by negative valence and low arousal). Here, we have performed an additional analysis to determine if human participants are better able to distinguish affective hand displays in this lower dimensional space of valence and arousal. Ekman emotion coordinates in arousal and valence dimensions taken from [39] were weighted by participants' Ekman emotion ratings for each affective hand movement, as shown in Equation (1). Coefficients $a, b, c, d, e$, and $f$ are the arousal $(H=$ Arousal $)$ or valence $(H=$ Valence $)$ coordinates of anger, disgust, fear, happy, sad and surprise emotions obtained from [38]. Emotion $i$ indicates the emotion (anger, disgust, fear, happiness, sadness, and surprise) rating from subject $i$. The results of the computed arousal ratings for each animation are shown in Figures 7 and 8.

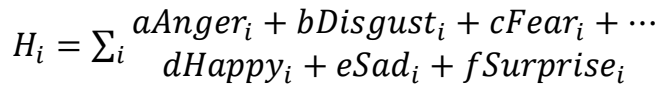

This mapping between the Ekman emotion ratings from this study and the valence-arousal model, results in similar arousal responses for Ekman emotions as those reported in [38]. The results show high arousal values for anger, medium arousal values for happiness, and low arousal values for sad movements. Subjects were able to distinguish differing levels of arousal in all three displays.

Arousal results were consistent regardless of the structure. However, the values obtained for the valence element of different affective movements were not consistent between subjects and not comparable to the corresponding valence coordinate for Ekman emotions reported in [38]. A tendency toward negative valence was observed for the valence values obtained here. Considering the formulation (Equation (1)) used in this study to obtain the valence associated with different affective movements, it is likely that the prevalence of negative emotions in the 6 basic Ekman emotions results in a skew towards negative valence values.

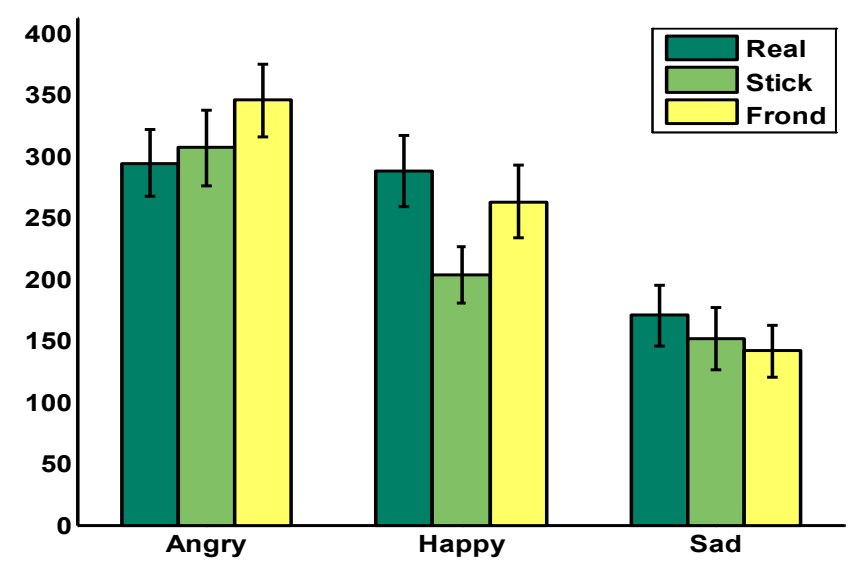

Fig. 7. Average arousal $(m e a n \pm S E)$ values for expressions displayed on different structures.

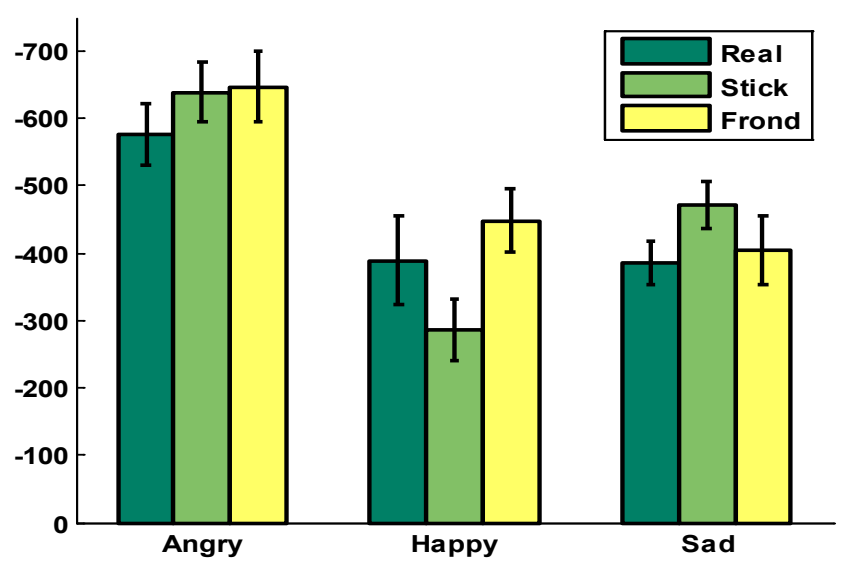

Fig. 8. Average valence (mean $\pm \mathrm{SE}$ ) values for expressions displayed on different structures.

We also carried out an ANOVA with the main effect of structure and the intended-emotion along with their interaction on the valence and arousal level evoked by observing different affective hand movements. Similar to the discrete emotion category analysis, the intended-emotion has a main effect on the valence and arousal values with no main affect of the moving structure as well as no interaction between the structure and the intended-emotion.

We have also performed pairwise hypothesis analysis (student $\mathrm{t}$-test) to check if the differences between the arousal level of the observed Ekman emotions are significant at $p<0.05$. We have found that the difference between arousal levels of the angry and sad hand movements is significant in all instances regardless of the structure (e.g., arousal value associated with subjects rating for Real_Angry movement is significantly different from sad movements displayed on the real, stick and frond structures). The pairwise differences between arousal values associated with the Real_Happy or Frond_Happy and those associated with sad movements displayed on different structures are significant. However, the difference between the Stick_Happy arousal and the arousal associated with the sad movement displayed on different structures was not found to be significant. Moreover, other than the happy movement displayed on the stick structure, there is no significant 
difference between the arousal values of Real_Happy or Frond_Happy and arousal values of the Real_Angry, Stick_Angry and Frond_Angry. This result is in line with the arousal values for happy and angry emotions reported in [39], which attributes positive arousal values to both angry and happy emotions.

\section{DISCUSSION}

Participants showed some ability to recognize emotions displayed on different hand-like structures: a human-like hand, a stick hand model and a frond structure. The angry emotion is recognized correctly regardless of the structure used to display the emotion. This result is consistent with previous work [11], [36], [37], reporting angry to be the most reliably identified emotion. Misrecognition of the happy expressive movements as angry was observed in the Frond_Happy animations. This is probably due to the similar nature of the movements associated with the happiness and anger emotions. In [8], both the happiness and the anger emotions are associated with movements with frequent tempo changes, similar movement kinematics and dynamic tensions. The ANOVA findings of main effect of intendedemotion and no main effect of structure on the perception of affective movements are in line with previous findings [42]. This study reports that motion perception is strongly influenced by motion characteristics (e.g. acceleration and curvature), while the type of embodiment has no effect on motion perception. While [42] studies the effect of the type of embodiment for different non-articulated structures, in our study the impact of physical appearance of an articulated hand-like structure on movement perception is investigated.

The results of the valence-arousal analysis of the emotion ratings suggest that the subjects were able to consistently recognize the arousal level associated with different affective movements regardless of the display structure. Further investigation is needed to evaluate human perception in recognizing the valence and arousal elements of affective movement and to identify the movement features associated with these elements (e.g. the effect of high versus low tempo on valence-arousal perception of an affective movement). For this purpose, more user studies should be carried out with a larger set of affective movements, and with evaluation questions framed in a valence-arousal model. This would help to determine whether humans have the ability to recognize all 6 basic Ekman emotions from hand movements alone, or if only negative (anger, fear, disgust, sadness) versus positive emotions (happiness) or low arousal emotions (e.g., sadness) versus high arousal (e.g., anger) can be recognized. The other possibility is that only those movements that exhibit extreme movement qualities in terms of Laban effort component [30] can be recognized well.

Context plays an important role in human motion perception [7]. Therefore, a direction for future work is to investigate the effect of context on the perception of hand movement, such as an accompanying audio signal or speech, or additional objects in the scene depicting the hand movement. Another issue is the impact of culture on the perception of gestural hand movements. For instance, in some cultures a fist hand posture represents aggressiveness and anger. Therefore, this culturally developed perception should be taken into consideration in designing studies exploring the human perception of gestural movements. In future studies, we will consider incorporating arm along with hand movements. Furthermore, the most representative features associated with different classes of emotional movements (in terms of expressiveness, and movement dynamics and kinematics) displayed on human-like and nonhuman-like structures will be investigated.

\section{CONCLUSION}

Human perception of expressive hand movements was examined through a user study. A series of expressive hand movements were performed, recorded and replayed on three different structures: a human-like hand model, a stick hand model and a frond like model. An online questionnaire containing animations of these expressive movements on the differing structures was used to evaluate human perception of the intended-emotions in these movements. The participants were somewhat effective at identifying the emotional categories conveyed by expressive hand movements displayed on different structures. Anger is the most correctly identified emotion regardless of the physical appearance of the display structure. For correctly perceived affect, the physical appearance of the structure on which the movements were displayed did not affect the perception of the conveyed affect. Valence-arousal analysis of participants' responses shows that subjects are able to consistently recognize various levels of arousal associated with different affective hand movements, while no consistent recognition of valence was observed. This result is promising for non-anthropomorphic structures intended to display affective movements (e.g., Hylozoic environments) as human perception of movement does not appear to be influenced by the physical appearance of the moving structure. However, further investigation is needed to determine the generalizability of this result to a wider range of human affective movements and to non-anthropomorphic structures.

\section{REFERENCES}

[1] C. Breazeal, "Designing Sociable Robots. Cambridge," MA: MIT Press, 2002.

[2] C. Breazeal, "Emotion and sociable humanoid robots.International Journal of Human Computer Studies", 59, 2003, pp. 119-155.

[3] B. Allison, G. Nejat, and E. Kao, “The Design of an Expressive Human-like Socially Assistive Robot," ASME Journal on Mechanisms and Robotics, Vol. 1, Iss. 1, 2009.

[4] T. Jansen, "Theo Jansen: The Great Pretender," Rotterdam: 010 Uitgeverij, 2007.

[5] M.R. Thomsen, "Metabolistic architecture. in Responsive Textile Environments," (S. Bonnemaison and C. Macy, eds.), Halifax: Tuns Press, 2007.

[6] S. Damiani, E. Deregibus, and L. Andreone,. "Driver-vehicle interfaces and interaction: where are they going?," European Conference of Transport Research Institutes (ECTRI), 2009. 
[7] R. Blake, and M. Shiffrar, "Perception of human motion," Annual Review of Psychology, 58, 12.1-12.27, 2007.

[8] A. Camurri, I. Lagerlöf , and G. Volpe, "Recognizing Emotion from Dance Movement: Comparison of Spectator Recognition and Automated Techniques," International Journal of Human-Computer Studies, 59(1-2), 2003, pp213-225.

[9] R. McDonnell, S. Jörg, J. McHugh, F. Newell, and C. O'Sullivan, "Evaluating the emotional content of human motions on real and virtual characters," Proceedings of the 5th Symposium on Applied Perception in Graphics and Visualization, APGV '08, ACM, New York, 2008, pp 67-74.

[10] M. Coulson, "Attributing emotion to static body postures: Recognition accuracy, confusions, and viewpoint dependence," Journal of Nonverbal Behavior 28, 2, 2004, pp117-139.

[11] H.G. Wallbott, 1998. Bodily expression of emotion. European Journal of Social Psychology 28, 6, 879-896.

[12] Z. Zeng, M. Pantic, G.I. Rosiman, and T.S. Huang, "A survey of affect recognition methods: Audio, visual, and spontaneous expressions," Trans. on Pattern Analysis and Machine Intelligence, vol. 31, no. 1, 2009, pp. 39-58.

[13] P. Ekman, "Are there basic emotions? Psychological Review," 99, 1992, pp 550-553.

[14] J.K. Hodgins, J.F. O'Brien, and J. Tumblin, "Perception of human motion with different geometrical models, " IEEE Trans. Vis. Comput. Graph, no. 4, 1998, pp 307-17.

[15] S. Jörg, J. Hodgins, C. O'Sullivan, "The perception of finger motions," Proceedings of the 7th Symposium on Applied Perception in Graphics and Visualization, Los Angeles, 2010, pp 129-133.

[16] D. Perani, F. Fazio, N.A. Borghese, M. Tettamanti, S. Ferrari, J. Decety, and M.C. Gilardi, "Different brain correlates for watching real and virtual hand actions, "Neuroimage 14, 2001, pp 749-758.

[17] S. Han, Y. Jinag, G.W. Humphreys, T. Zhou, and P. Cai, "Distinct neural substrates for the perception of real and virtual visual worlds," NeuroImage, 24, 2005, pp 928-935.

[18] R.A. Mar, W.M. Kelley, T.F. Heatherton, and C.N. Macrae, "Detecting agency from the biological motion of veridical vs. animated agents," Social Cognitive and Affective Neuroscience, no. 2, 2007, pp 199-20.

[19] T. Shibata, K.Wada, and K. Tanie, "Subjective evaluation of a seal robot at the national museum of science and technology in Stockholm," In proceedings of the $12^{\text {th }}$ IEEE international workshop on Robot and Human Interactive, 2003, pp 397-402.

[20] C. Breazeal, and B. Scassellati, "How to build robots that make friends and influence people," In proceedings of the International Conference on Intelligent Robots and Systems, Kyongjo, Korea, 1999.

[21] J.H. Oh, D. Hanson, W.S. Kim, I.Y. Han, J.Y. Kim and I.W. Park, "Design of Android type Humanoid Robot Albert HUBO," IEEE International Conference on Intelligent Robots and Systems, Beijing, Oct. 2006, pp 1428-1433

[22] J. Tegin, and J. Wikander, "Tactile sensing in intelligent robotic manipulation: a review," Industrial Robot: An International Journal, vol. 32, no. 1, 2005, pp 64-70.

[23] M. Wanderley, and P. Dapalle, "Gestural control of sound synthesis," In proceedings of the IEEE, vol. 92, no.4. 2004, pp 632-644.

[24] R. Cowie, "Perceiving emotions: towards a realistic understanding of the task," Philosophical Transactions of the Royal Society of London. Series B, Biological Sciences, vol. 364, no. 15235, 2009, pp 35153525.

[25] R. Laban, and F.C.L. Lawrence, "Effort," London, Macdonald \& Evans, 1947.

[26] D. Winter, "The Biomechanical and Motor Control of Human Movement," second ed. John Wiley \& Sons, 1990.

[27] R. Laban. "The Mastery of Movement," MacDonald and Evans, 1963.

[28] S. Zhang, Q. Li, T. Yu, X. Shen, W. Geng, and P. Wang, "Implementation of a notation-based motion choreography system," interactive Technologies and Sociotechnical Systems, no. 4270 in Lecture notes in Computer Science, Berlin: Springer, 2006, pp 495503.

[29] K. Hachimura, K. Takashina, and M. Yoshimura, "Analysis and Evaluation of Dancing Movement Based on LMA, " IEEE International Workshop on Robot and Human Interactive Communication, 2005.

[30] J. Rett, J. Ahuactzin, and J. Dias, "Bayesian reasoning for LABAN Movement Analysis used in Human Machine Interaction,"
International Journal of Reasoning-based Intelligent Systems (IJRIS), 2008.

[31] F.E. Pollick, H.M. Paterson, A. Bruderlin, and A.J. Sanford, "Perceiving affect from arm movement," Cognition 82, B51-B61, 2001.

[32] P. Beesley, "Kinetic Architectures and Geotextile Installations," Toronto: Riverside Architectural Press, 2010.

[33] P. Beesley, "Hylozoic Soil," Leonardo, Vol. 42, no. 4, 2009, pp 360361.

[34] "Measurand," http://www.measurand.com/

[35] "BioVision Hierarchy", http://www.BioVision.com/

[36] R.D. Walk, and C. P. Homan, "Emotion and dance in dynamic light displays, " Bulletin of the Psychonomic Society, 22, 1984, pp 437440.

[37] W.H. Dittrich, T. Troscianko, S.E.G. Lea, and D. Morgan, "Perception of emotion from dynamic point-light displays represented in dance," Perception, 25, 1996, pp 727-738.

[38] J.A. Russell, "A circumplex model of affect," Journal of Personality and Social Psychology, no. 39, 1980, pp 1161-1178.

[39] R.J. Larsen, and E. Diener, "Promises and problems with the circumplex model of emotion," In M. S. Clark (Ed.), Review of personality and social psychology. Vol. 13, 1992, pp. 25-59.

[40] H. Schlosberg, "The description of facial expressions in terms of two dimensions," Journal of Experimental Psychology, no. 44, 1952, pp 229-237.

[41] G. Lu, L. Shark, G. Hall and U. Zeshan, "Dynamic Hand Gesture Tracking and Recognition for Real-Time Immersive Virtual Object Manipulation", International Conference on CyberWorlds, 2009, pp 29-35.

[42] M. Saerbeck and C. Bartneck, "Perception of affect elicited by robot motion," in International Conference on Human-Robot Interaction, Osaka 2010, pp. 53-60.

[43] R. M. Krauss and U. Hadar, "The role of speech-related arm/hand gestures in word retrieval," Gesture, speech, and sign, pp. 93-116, 1999.

[44] A.-A. Samadani. Survey Videos. Available: https://ece.uwaterloo.ca/ asamadan/surveyVideos.htm

[45] J. K. Hietanen, J. M. Leppänen, and U. Lehtonen, "Perception of emotions in the hand movement quality of Finnish sign language," Journal of nonverbal behavior, vol. 28, pp. 53-64, 2004. 